\title{
THE NATURE OF EQUILIBRIUM IN A LOCATION MODEL*
}

\author{
By Martin J. Osborne and Carolyn Pitchik**
}

\section{INTRODUCTION}

Models of location are appropriate in a number of contexts in economics and political science. For example, firms choose where to position stores and which of a spectrum of goods to produce, and politicians select the nature of their platforms. In such models it is natural to look for a collection of locations with the property that the location of each individual is optimal, given the positions of all other individuals. However, the pure strategy Nash equilibrium provides a solution which is both incomplete and unsatisfactory. Incomplete, because in many cases no such equilibrium exists. Unsatisfactory, because even when it does exist it may not be robust to the specification of the model.

Consider, for example, a simple case (the "pure" location model of Hotelling [1929]). Consumers are distributed on the interval [0,1]. Each of a fixed number of firms chooses a location in $[0,1]$ and receives a payoff equal to the fraction of consumers for which it is the nearest firm. Then if there are three firms, there is no pure strategy equilibrium unless the distribution of consumers is degenerate. There is such an equilibrium if there are four or more firms and the distribution of consumers is uniform; but there is none if there are five or more firms and the density of this distribution is either strictly convex or strictly concave, however close it is to being uniform.

One way to avoid these problems is to modify the model. For example, if the firms locate on a circle rather than a line segment, or move sequentially rather than simultaneously, the difficulties may be mitigated. However, in many cases of interest a line segment is the relevant space, and the assumption of simultaneous moves is appropriate (or, at least, any particular sequence of moves is arbitrary).

For these reasons, our approach is to study a modification of the solution. The standard game theoretic route in the absence of pure strategy equilibria is to allow the players to randomize. Given that many location decisions are more or less irreversible, this solution makes sense (if there is ex post mobility then the

* Manuscript received July, 1982; revised August, 1985.

** We are grateful to Roger Myerson and two referees for very helpful comments and suggestions. This work was begun when Pitchik was visiting the Cowles Foundation for Research in Economics at Yale University in 1980-81. She is grateful for its hospitality, and for support from a Post-Doctoral Fellowship from the Natural Sciences and Engineering Research Council, Canada, and from a grant from the Office of Naval Research (contract N00014-77-C-0518). Her work was also partially supported by grants from the Office of Naval Research (contract N0014-78-C-0598) and National Science Foundation (SES-8207765) at New York University. Osborne's work was partially supported by grants from the Council for Research in the Social Sciences at Columbia University, and from the National Science Foundation (SES-8318978 and SES-8510800). 
appropriate model is a repeated game). However, the idea of individuals consciously randomizing may be unappealing. If so, the results on the "purification" of equilibria provide an attractive alternative interpretation. The basic idea is that a mixed strategy equilibrium can, under appropriate conditions, be viewed as a pure strategy equilibrium in a game of incomplete information. Firms may not know precisely what the payoffs are - for example, they may obtain information about the distribution of consumers from noisy market surveys. If the private signals they receive are independent and atomless, then an equilibrium in which firms randomize may be equivalent to one in which, contingent on the signal received, each firm uses a pure strategy. The randomness of the signals means that the action taken by each firm varies randomly, but no firm consciously randomizes.

A variety of results on purification has been established, notably by Aumann et al. [1983] and Milgrom and Weber [1981]. Our games do not satisfy the assumptions in either of these papers, since each firm has a continuum of pure strategies (rather than finitely many, as in Aumann et al.), and its payoff function has discontinuities (rather than being continuous, as (for the most part) in Milgrom and Weber). Thus we cannot directly apply their results; it is possible that the results could be extended to cover our games. The type of purification result of perhaps the most relevance to our games is the convergence result of Harsanyi [1973] (although once again our games do not satisfy his assumptions). He considers approximating a game of complete information with a sequence of games of incomplete information. Each of the latter has a pure strategy equilibrium, as described above, in which the action of each player depends on his type, and is thus random from the point of view of the other players. Harsanyi shows that pure strategy equilibria in a sequence of approximating games can almost always be found with the property that the distributions over pure strategies which these equilibria generate converge to the distribution associated with any given mixed strategy equilibrium in the original game. In our context, games in which the firms are slightly uncertain about their payoffs may have equilibria which generate distributions over actions which are very similar to those generated by the mixed strategy equilibria we find. (Section 5 of Milgrom and Weber [1981], and Harsanyi [1977] contain further discussion of issues in the interpretation of mixed strategy equilibria.)

Our results concern mixed strategy equilibrium in the simple location model described above. They are of three types. First, we study the symmetric mixed strategy equilibria $(F, \ldots, F)$ for an arbitrary distribution of consumers and arbitrary number of firms (see Proposition 3). An explicit characterization of an equilibrium strategy $F$ appears to be impossible; we show that any such $F$ possesses some natural properties. Second, we show that the symmetric equilibrium strategy approaches the distribution function of consumers as the number of firms increases (see Proposition 4). That is, when the number of firms is large, the firms distribute themselves in the same way as the consumers. This makes sense: when there are many firms, it is likely that each firm will have 
neighbors to the left and right, so that the endpoints of the line segment exert little influence on the solution, and firms spread out according to the distribution of consumers, as they would on a circle. Finally, we study the case of three firms and a uniform distribution of consumers. It is known that in the symmetric mixed strategy equilibrium of this game, each firm randomizes uniformly over $[1 / 4,3 / 4]$ (see Shaked [1982]). We show that the game has other, asymmetric equilibria. In fact, there is a unique (up to symmetry) equilibrium in which at least one firm uses a pure strategy (see Propostion 5). In this equilibrium, two firms randomize, putting most weight near $1 / 4$ and $3 / 4$ (see Figure 1), while the third locates with probability one at $1 / 2$.

Our results on the qualitative properties of equilibrium have direct empirical implications. For example, across independent three-person political races, the prediction is that either all the platforms chosen will be uniformly distributed over the middle two quartiles of the spectrum, or one will always be in the center, while the other two are close to the quartiles.

\section{THE MODEL AND RESULTS}

Consumers are distributed on the line segment $[0,1]$. Let $C(x)$ be the fraction of consumers to the left of $x$ plus half of the fraction at $x$ (so that if there are no atoms in the distribution of consumers, $C$ is just the cumulative distribution function), Let $\Gamma^{n}(C)$ be the game in which $n$ firms choose locations in $[0,1]$. Precisely, the pure strategy set of each firm is $S=[0,1]$, and each payoff function $K_{i}: S^{n} \rightarrow \boldsymbol{R}$ is defined as follows. Let $s_{\sim}=\left(s_{1}, \ldots, s_{n}\right) \in S^{n}$, let $L_{i}\left(s_{\sim}\right)$ and $R_{i}\left(s_{\sim}\right)$ be the sets of firms to the left and right of $i$ in $s$, let $l_{i}(s)$ and $r_{i}(s)$ be the positions of the firms in $L_{i}(s)$ and $R_{i}(s)$ closest to $i$, let $\lambda_{i}(s)=\left(s_{i}+l_{i}(s)\right) / 2$ and $\rho_{i}\left(s_{\sim}\right)=\left(s_{i}+r_{i}(s)\right) / 2$ (the locations of the consumers equidistant from $i$ and its nearest neighbors), and let $q_{i}(s)$ be the number of firms located at $s_{i}$ in $s_{\sim}$. Then

$$
K_{i}(s)= \begin{cases}{\left[C\left(\rho_{i}(s)\right)-C\left(\lambda_{i}(s)\right)\right] / q_{i}(s)} & \text { if } \quad L_{i}(s) \neq \phi, R_{i}(s) \neq \phi \\ C\left(\rho_{i}(s)\right) / q_{i}(s) & \text { if } \quad L_{i}(s)=\phi, R_{i}(s) \neq \phi \\ {\left[1-C\left(\lambda_{i}(s)\right)\right] / q_{i}(s)} & \text { if } \quad L_{i}(s) \neq \phi, R_{i}(s)=\phi \\ 1 / q_{i}(s) & \text { if } \quad L_{i}(s)=\phi, R_{i}(s)=\phi .\end{cases}
$$

As usual, the set of mixed strategies of each firm is the set of cumulative distribution functions on $S$, and we extend $K_{i}$ to mixed strategy $n$-tuples. We always use upper case letters (e.g. $F, G$ ) to denote mixed strategies, reserving lower case letters for pure strategies. Thus when, for example, we write $K_{i}(s, F, t)$, it is to be understood that firms 1 and 3 are using pure strategies, and firm 2 is using a mixed strategy.

The two-firm game $\Gamma^{2}(C)$ can easily be completely solved. Call $m$ a median of $C$ if $C(m-) \leqq 1 / 2 \leqq C(m+)$ (where $C(m-)$ and $C(m+)$ are the left and right limits of $C$ at $m$ ); the set $M(C)$ of medians of $C$ is a nonempty closed interval. 
It is clear that the strategy pair $\left(F_{1}, F_{2}\right)$ is an equilibrium of $\Gamma^{2}(C)$ if the support of each $F_{i}$ (denoted supp $F_{i}$ ) is a subset of $M(C)$. It is also easy to show that there are no other equilibria, as follows.

Proposition 1. The strategy pair $\left(F_{1}, F_{2}\right)$ is an equilibrium of $\Gamma^{2}(C)$ if and only if supp $F_{i} \subseteq M(C)$ for $i=1,2$. In particular, if $C$ has a unique median $m$ then $\Gamma^{2}(C)$ has a unique equilibrium, in which each firm locates at $m$ with probability one.

Proof. It is clear that $\left(s_{1}, s_{2}\right)$ is a pure strategy equilibrium if $s_{i} \in M(C)$ for $i=1,2$. Suppose $\left(F_{1}, F_{2}\right)$ is also an equilibrium. Then since $\Gamma^{2}(C)$ is constant sum, $\left(F_{1}, s_{2}\right)$ is an equilibrium. But this is so only if supp $F_{1} \subseteq M(C)$.

Lerner and Singer [1937] and Eaton and Lipsey [1975] have studied the pure equilibria of $\Gamma^{n}(C)$ for $n \geqq 3$. Eaton and Lipsey show, in particular, the following. ${ }^{1}$

Proposition 2. (Eaton and Lipsey). (a) If $C$ is increasing and $\left(s_{1}, \ldots, s_{n}\right.$ ) is a pure strategy equilibrium of $\Gamma^{n}(C)$ with $s_{1} \leqq \cdots \leqq s_{n}$ then $s_{1}=s_{2}$ and $s_{n-1}=s_{n}$.

(b) If $C$ is increasing then $\Gamma^{3}(C)$ has no pure strategy equilibrium.

(c) If $C$ is differentiable then $\Gamma^{n}(C)$ has a pure strategy equilibrium only if $n$ is at most twice the number of local maximizers of $C^{\prime}$.

(d) If $C$ is uniform then $\Gamma^{\prime \prime}(C)$ possesses a unique pure strategy equilibrium if $n=4$ or 5 , and a continuum of such equilibria if $n \geqq 6$.

Part (a) of this result is easy to see: if a peripheral firm is not paired then since $C$ is increasing, the firm can gain customers by moving towards its neighbor. Part (b) follows from (a), given that $s_{1}=s_{2}=s_{3}$ is obviously not an equilibrium. The equilibria in (d) are easy to find. For example, if $n=4$ then $s_{1}=s_{2}=1 / 4, s_{3}=s_{4}=$ $3 / 4$ defines the equilibrium, and if $n=5$ we have $s_{1}=s_{2}=1 / 6, s_{3}=1 / 2$, and $s_{4}=$ $s_{5}=5 / 6$.

Part (b) shows that pure strategy equilibrium is an inadequate solution concept for the location problems we are considering. The implications of part (c) emphasize this: if $C$ is differentiable and $C^{\prime}$ is either strictly concave (one local maximizer) or strictly convex (two local maximizers) then, however close $C$ is to being uniform, $\Gamma^{n}(C)$ possesses no pure strategy equilibrium if $n \geqq 5$. More generally, if $C^{\prime}$ has finitely many local maximizers then $\Gamma^{n}(C)$ possesses no pure strategy equilibrium when the number of firms is large enough.

Thus the pure strategy equilibria are nonrobust to variations in the specification of the model; our approach is to turn to the mixed strategy equilibria. We prove three results about these equilibria. It is known from the work of Dasgupta and Maskin [1982a and b] that $\Gamma^{n}(C)$ possesses a symmetric mixed strategy equi-

\footnotetext{
Note that " $n \geqq 2 M^{\prime \prime}$ about two thirds of the way down their p. 35 is a typographical error for " $n \leqq 2 M "$.
} 
librium $\left(F_{C}^{n}, \ldots, F_{C}^{n}\right)$. (See also Simon [1984] for a very general existence theorem for equilibria in location games.) We first study the general characteristics of $F_{C}^{n}$, and then prove a result on its asymptotic behavior as $n \rightarrow \infty$. Finally, when $n=3$ and $C$ is uniform we find all (asymmetric) equilibria in which at least one firm uses a pure strategy. All proofs are in the Appendix.

Our first result is the following.

Proposition 3. If $n \geqq 3$ and $C$ is nonatomic then the game $\Gamma^{n}(C)$ has a symmetric mixed strategy equilibrium $\left(F_{C}^{n}, \ldots, F_{C}^{n}\right)$, and in every such equilibrium $F_{C}^{n}$ is atomless. If $C$ is symmetric about $1 / 2$ (i.e. $C(s)=1-C(1-s)$ ) then $\Gamma^{n}(C)$ has an atomless symmetric mixed strategy equilibrium $\left(F_{C}^{n}, \ldots, F_{C}^{n}\right)$ in which $F_{C}^{n}$ is symmetric about $1 / 2$. If $C$ is uniform then in addition the support of $F_{C}^{n}$ is an interval $\left[a_{C}^{n}, 1-a_{C}^{n}\right]$.

Even though Proposition 3 puts some restrictions on the characteristics of a symmetric equilibrium, it would be nice to have some explicit examples. However, except in the case $n=3$ and $C$ uniform, it seems not to be possible to make the necessary calculations. To appreciate the problem, consider the payoff of firm 1 when it locates at $z$ and every other firm uses the atomless strategy $F$. This payoff is

$$
\begin{aligned}
K_{1}(z, F, \ldots, F)= & (n-1) \int_{0}^{z}[1-C((u+z) / 2)](F(u))^{n-2} d F(u) \\
& +\sum_{k=0}^{n-3}(n-1)(n-2)\left(\begin{array}{c}
n-3 \\
k
\end{array}\right) \int_{0}^{z} \int_{z}^{1}[C((z+v) / 2) \\
& -C((z+u) / 2)](F(u))^{k}(1-F(v))^{n-k-3} d F(v) d F(u) \\
& +(n-1) \int_{z}^{1} C((u+z) / 2)(1-F(u))^{n-2} d F(u) .
\end{aligned}
$$

Using the binomial theorem on the middle term, and integrating by parts, this reduces, in the case where $C$ is differentiable, to

$$
\begin{aligned}
K_{1}(z, F, \ldots, F)= & {[1-C((1+z) / 2)](F(z))^{n-1}+C(z / 2)(1-F(z))^{n-1} } \\
& +(1 / 2) \int_{0}^{z} C^{\prime}((u+z) / 2)(1-F(z)+F(u))^{n-1} d u \\
& +(1 / 2) \int_{z}^{1} C^{\prime}((u+z) / 2)(1+F(z)-F(u))^{n-1} d u .
\end{aligned}
$$

The problem of finding a symmetric equilibrium is thus the problem of finding a nondecreasing $F$ such that $K_{1}(z, F, \ldots, F)$ is constant on supp $F$. Even if $C$ is uniform this is a difficult problem - (2) is a nonlinear integral equation, about which little in general is known.

We can however use (2) to prove a result on the behavior of $F$ as $n$ increases without bound. The idea is simple. If there is a large number of firms, all using $F$, then wherever firm $i$ locates it is very likely to have neighbors close to the left 
and right, and we can ignore the possibility that all the other firms are on one side of firm $i$. If $C$ is uniform but $F$ is not, then at those points where the density of $F$ exceeds 1 , the left and right neighbors are likely to be closer than when this density is less than 1, so that the payoff at the former is lower than at the latter. Thus such an $F$ cannot be an equilibrium - a firm is not indifferent between all points in the support of $F$. Similarly, for arbitrary (but differentiable) $C$, when $n$ is large the payoff is not constant if $F^{\prime}(x)$ is different from $C^{\prime}(x)$. Formally, the result is as follows. (The strong assumptions, which make the proof relatively straightforward, can probably be relaxed somewhat.)

Propositron 4. Let $C$ be twice continuously differentiable and suppose $\left(F^{n}, \ldots, F^{n}\right)$ is an equilibrium of $\Gamma^{n}(C)$ for each $n=1,2, \ldots$, where $F^{n}$ is twice continuously differentiable and there exists a strategy $F$ which is twice continuously differentiable such that $F^{n} \rightarrow F, F^{\prime \prime} \rightarrow F^{\prime}$, and $F^{\prime \prime \prime} \rightarrow F^{\prime \prime}$ uniformly. Then $F=C$.

When $n=3$ and $C$ is uniform, it is known that there is a symmetric equilibrium $(F, F, F)$ in which $F$ is uniform on $[1 / 4,3 / 4]$ (see Shaked [1982]). Our final result exhibits all the asymmetric equilibria, within a certain class, of this game. Let $U$ be the uniform distribution.

Proposition 5. There is a unique (up to symmetry) equilibrium of $\Gamma^{3}(U)$ in which at least one firm uses a pure strategy. It is $(1 / 2, F, F)$, where the support of $F$ is $[5 / 24,19 / 24]$ and

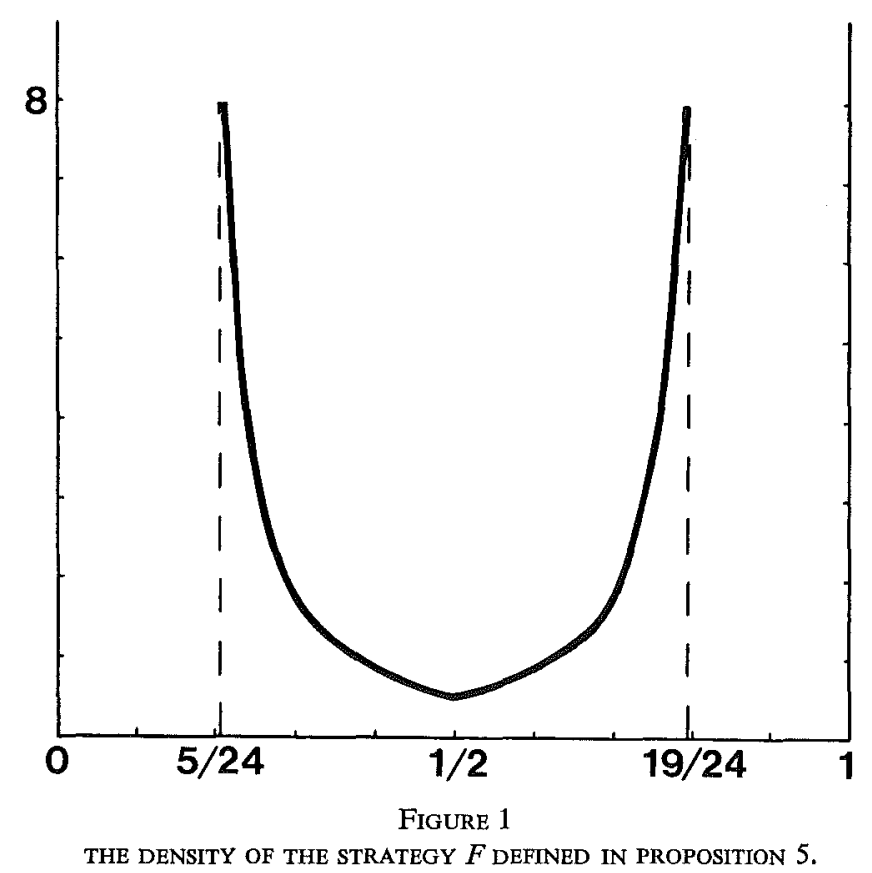




$$
F(t)= \begin{cases}1-2^{-2 / 3}(6 t-1)^{-1 / 3} & \text { if } \quad 5 / 24 \leqq t \leqq 1 / 2 \\ 2^{-2 / 3}(5-6 t)^{-1 / 3} & \text { if } \quad 1 / 2 \leqq t \leqq 19 / 24\end{cases}
$$

The corresponding equilibrum payoffs are (38/96, 29/96, 29/96).

The density of the strategy $F$ given in this result is illustrated in Figure 1. Note that the result does not rule out the possibility of asymmetric mixed strategy equilibria in which all three firms randomize.

Columbia University, U.S. A.

and McMaster University, Canada

University of Toronto, Canada

APPENDIX: PROOFS

We use the following notation. For any strategy $F, \alpha(F)$ and $\beta(F)$ are respectively the smallest and largest members of supp $F, J(F)$ is the set of atoms of $F$, and $J_{F}(x)$ is the size of the atom $x$ of $F$. For any $n$-tuple $F=\left(F_{1}, \ldots, F_{n}\right)$ of strategies and any strategy $G$ of firm $i, P_{i}(G, \underset{\sim}{F})=K_{i}\left(F_{1}, \ldots, F_{i-1}, G, F_{i+1}, \ldots, F_{n}\right)$. For any function $f, f(x-)$ and $f(x+)$ are respectively the left and right limits of $f$ at $x$.

The following result (a generalization of Proposition 2(b)) is used in the proofs of Propositions 3 and 5.

LEMMA 1. Let $C$ be atomless. Then in any equilibrium of $\Gamma^{n}(C)$, each point in $[0,1]$ is an atom of the strategies of at most two firms.

Proof, Let $F=\left(F_{1}, \ldots, F_{n}\right)$ be an equilibrium of $\Gamma^{n}(C)$. If $x$ is an atom of $F_{1}$ then we need $P_{1}(x, F) \geqq P_{1}(x-, F)$ and $P_{1}(x, F) \geqq P_{1}(x+, F)$, and hence, in particular, we need

$$
\left[P_{1}(x, F)-P_{1}(x-, \underset{\sim}{F})\right]+\left[P_{1}(x, \underset{\sim}{F})-P_{1}(x+, \underset{\sim}{F})\right] \geqq 0,
$$

or, using the Dominated Convergence Theorem and letting $s_{\sim}=\left(x, s_{2}, \ldots, s_{n}\right)$,

$$
\begin{aligned}
& \int_{0}^{1} \cdots \int_{0}^{1}\left(\left[P_{1}(x, \underset{\sim}{s})-P_{1}(x-, s)\right]\right. \\
& \left.\quad+\left[P_{1}(x, s)-P_{1}(x+, s)\right]\right) d F_{2}\left(s_{2}\right) \cdots d F_{n}\left(s_{n}\right) \geqq 0 .
\end{aligned}
$$

Now, using (1) and the nonatomicity of $C$, we find that

$$
\begin{aligned}
& {\left[P_{1}(x, \underset{\sim}{s})-P_{1}(x-, s)\right]+\left[P_{1}(x, s)-P_{1}(x+, s)\right] } \\
& \quad= \begin{cases}0 & \text { if } q_{1}(s)=1 \\
\left(2 / q_{1}(s)-1\right)\left(C\left(\hat{\rho}_{1}(s)\right)-C\left(\hat{\lambda}_{1}(s)\right)\right) & \text { if } q_{1}(s) \geqq 2,\end{cases}
\end{aligned}
$$

where $\hat{\rho}_{1}(s)=\rho_{1}(s)$ if $R_{1}(\underline{\sim}) \neq \phi, \hat{\rho}_{1}(\underline{\sim})=1$ if $R_{1}(\underset{\sim}{s})=\phi$, and $\hat{\lambda}_{1}(s)=\lambda_{1}(s)$ if $L_{1}(s)$ 
$\neq \phi, \hat{\lambda}_{1}(\underbrace{}_{\sim})=0$ if $L_{1}(s)=\phi . \quad$ Since $\hat{\rho}_{1}(s) \geqq \hat{\lambda}_{1}\left(s_{\sim}\right)$ for all $\left(s_{2}, \ldots, s_{n}\right)$, we have $C\left(\hat{\rho}_{1}(s)\right)$ $\geqq C\left(\hat{\lambda}_{1}(s)\right)$ for all $\left(s_{2}, \ldots, s_{n}\right)$. Thus, using (4), the integrand in the left-hand side of (3) is nonpositive for all $\left(s_{2}, \ldots, s_{n}\right)$; because of the inequality in (3) it must therefore be zero except possibly on a set of $F_{2} \cdots F_{n}$-measure zero. Hence if the strategies of two firms - say firms 2 and 3 - in addition to firm 1 have atoms at $x$, then, since $2 / q_{1}\left(x, x, x, s_{4}, \ldots, s_{n}\right)-1<0$ for all $s_{4}, \ldots, s_{n}$, we need $C\left(\hat{\rho}_{1}\left(x, x, x, s_{4}, \ldots, s_{n}\right)\right)=$ $C\left(\hat{\lambda}_{1}\left(x, x, x, s_{4}, \ldots, s_{n}\right)\right)$ for all $\left(s_{4}, \ldots, s_{n}\right)$ except possibly a set of $F_{4} \cdots F_{n}$-measure zero. Since $C\left(\hat{\rho}_{1}\left(x, x, x, s_{4}, \ldots, s_{n}\right)\right) \geqq C\left(\hat{\rho}_{1}\left(x, s_{2}, \ldots, s_{n}\right)\right)$ and $C\left(\hat{\lambda}_{1}\left(x ; x, x, s_{4}, \ldots, s_{n}\right)\right)$ $\leqq C\left(\hat{\lambda}_{1}\left(x, s_{2}, \ldots, s_{n}\right)\right)$ for all $\left(s_{2}, \ldots, s_{n}\right)$, this implies that $C\left(\hat{\rho}_{1}\left(x, s_{2}, \ldots, s_{n}\right)\right)=C\left(\hat{\lambda}_{1}(x\right.$, $\left.s_{2}, \ldots, s_{n}\right)$ ) for all $\left(s_{2}, \ldots, s_{n}\right)$ except possibly a set of $F_{2} \cdots F_{n}$-measure zero. (If, when firms 2 and 3 locate at $x$, firm 1 attracts no customers with probability one, then this is also true wherever firms 2 and 3 locate) But then $P_{1}(x, \underset{\sim}{F})=0$, contradicting the fact that the equilibrium payoff of firm 1 is positive (since it can obtain a positive payoff by locating at any point in the support of $C$ ). Thus the strategy of at most one firm in addition to firm 1 has an atom at $x$.

Proof of Proposition 3. If $n \geqq 3$ and $C$ is nonatomic then the game $\Gamma^{n}(C)$ possesses a symmetric mixed strategy equilibrium by Theorem 6 of Dasgupta and Maskin [1982b]. Let $(F, \ldots, F)$ be such an equilibrium. By Lemma $1, F$ is nonatomic.

We now show that if $C$ is symmetric about $1 / 2$ then $\Gamma^{n}(C)$ possesses an equilibrium $(F, \ldots, F)$ in which $F$ is symmetric about $1 / 2$. Consider the game $\bar{\Gamma}^{\prime \prime}(C)$ in which the pure strategy set $\bar{S}$ of each firm is $[0,1 / 2]$, and the payoff function of $i$ is defined by

$\bar{K}_{i}\left(\bar{s}_{1}, \ldots, \bar{s}_{n}\right)=K_{i}\left(1 / 2\left(\bar{s}_{1}\right) * 1 / 2\left(1-\bar{s}_{1}\right), \ldots, 1 / 2\left(\bar{s}_{n}\right) * 1 / 2\left(1-\bar{s}_{n}\right)\right)$ for $i=1, \ldots, n$,

where $1 / 2(x) * 1 / 2(1-x)$ denotes the mixed strategy in $\Gamma^{n}(C)$ which has atoms of size $1 / 2$ at $x$ and $1-x$. That is, using a pure strategy $\bar{s}$ in $\bar{\Gamma}^{\prime \prime}(C)$ is the same as using $\bar{s}$ with probability $1 / 2$ and $1-\bar{s}$ with probability $1 / 2$ in $\Gamma^{n}(C)$. Note that given the definition of $\bar{K}_{i}$, whenever $\bar{F}_{i}$ is a mixed strategy for each $i$ we have $\bar{K}_{i}\left(\bar{F}_{1}, \ldots, \bar{F}_{n}\right)=K_{i}\left(F_{1}, \ldots, F_{n}\right)$ where $F_{i}$ is defined by $F_{i}(x)=\bar{F}_{i}(x) / 2$ if $0 \leqq x<1 / 2$ and $F_{i}(x)=1-\bar{F}_{i}(1-x) / 2$ if $1 / 2 \leqq x \leqq 1$.

Now, by Theorem 6 of Dasgupta and Maskin [1982a] the game $\bar{\Gamma}^{n}(C)$ possesses a symmetric mixed strategy equilibrium $(\bar{F}, \ldots, \bar{F})$. Let $F$ be the mixed strategy corresponding to $\bar{F}$, as above. Then

$$
\dot{P}_{i}(\bar{s}, \bar{F}, \ldots, \bar{F}) \leqq \bar{K}_{i}(\bar{F}, \ldots, \bar{F})=K_{i}(F, \ldots, F) \quad \text { for all } \quad \bar{s} \in \bar{S} .
$$

But we have $\bar{P}_{i}(\bar{s}, \bar{F}, \ldots, \bar{F})=P_{i}(1 / 2(\bar{s}) * 1 / 2(1-\bar{s}), \quad F, \ldots, F)=P_{i}(\bar{s}, F, \ldots, F) / 2+$ $P_{i}(1-\bar{s}, F, \ldots, F) / 2=P_{i}(\bar{s}, F, \ldots, F)$, using the symmetry of $C$ and $F$ to obtain the last equality. Hence $(F, \ldots, F)$ is an equilibrium of $\Gamma^{n}(C)$ with $F$ symmetric about $1 / 2 ; F$ is atomless by the first part of the result.

Finally, we show that if $C$ is uniform and $(F, \ldots, F)$ is an equilibrium in which $F$ is symmetric about $1 / 2$ then the support of $F$ is an interval $[a, 1-a]$. Suppose, to the contrary, that $x \notin \operatorname{supp} F, a<x<1-a . \quad$ Let $b=\max \{s \leqq x: s \in \operatorname{supp} F\}$ and 
$c=\min \{s \geqq x: s \in \operatorname{supp} F\} ; b$ and $c$ exist since supp $F$ is closed, and $F(b)=F(c)$, since $F$ is nonatomic. Let $F=(F, \ldots, F)$. Then (dropping the superfluous subscript from $P_{i}(x, F)$ ) we have

$$
P(c, \underset{\sim}{F})-P(b, F)=\left[(1-F(b))^{n-1}-(F(b))^{n-1}\right](c-b) / 2 .
$$

$\left(P(c, s)-P(b, \underset{\sim}{s})\right.$ is zero unless either $s_{i}<b$ for all $i$ (in which case it is $\left.-(c-b) / 2\right)$, $s_{i}>c$ for all $i$ (in which case it is $(c-b) / 2$ ), or $s_{i}=b$ or $c$ for some $i$. The probabilities of these three events are $(F(b))^{n-1},(1-F(b))^{n-1}$ and zero respectively.) Since $F$ is atomless, we need $P(c, F)=P(b, \underset{\sim}{F})=1 / n$ (the equilibrium payoff of each firm). Hence, $F(b)=1 / 2$.

Since $F$ is nondecreasing and atomless, and $b \in \operatorname{supp} F, F$ is left-increasing at $b$. Therefore the support of $F$ contains at most a single gap. Since $F$ is symmetric, this means that $\operatorname{supp} F=[a, b] \cup[1-b, 1-a]$.

We now argue that $b=1 / 2$. In order for $F$ to be an equilibrium we need $P(z, F)=1 / n$ almost everywhere with respect to $F$. Now, for $0 \leqq z \leqq 1$ we have

$$
\begin{aligned}
& 2 P\left(z, F^{\prime}\right)=(a+1-z)(F(z))^{n-1}+(a+z)(1-F(z))^{n-1} \\
& \quad+\int_{a}^{z}(1-F(z)+F(u))^{n-1} d u+\int_{z}^{1-a}(1+F(z)-F(u))^{n-1} d u
\end{aligned}
$$

(see (2)). Thus, since $F$ is atomless, $P(\cdot, F)$ is continuous, and hence we need $P(z, F)=1 / n$ for all $z \in \operatorname{supp} F$. Thus $P(\cdot, F)$ is differentiable, with derivative zero, on $[a, b] \cup[1-b, 1-a]$. Now, if $F$ is differentiable at $z$ then so is $P(\cdot, F)$, and we have

$$
\begin{aligned}
& 2 P^{\prime}(z, F)=(n-1) F^{\prime}(z)\left\{(a+1-z)(F(z))^{n-2}-(a+z)(1-F(z))^{n-2}\right\} \\
& \quad+(n-1) F^{\prime}(z)\left\{\int_{a}^{1-z}(F(u)+F(z))^{n-2} d u-\int_{a}^{z}(F(u)+1-F(z))^{n-2} d u\right\} \\
& \quad+\left\{(1-F(z))^{n-1}-(F(z))^{n-1}\right\}
\end{aligned}
$$

where the first term in braces is obtained by using the symmetry of $F$. Suppose that $b<1 / 2$. Then for $z=b$ the first two terms in braces are positive and the third is zero (given $F(b)=1 / 2$ ). Hence given the continuity of $F$, the first two terms are positive on $(b-\varepsilon, b)$ for some $\varepsilon>0$; the third term is also positive on this interval. Further, since $F$ is almost everywhere differentiable (being a cumulative distribution function), it is differentiable somewhere in $(b-\varepsilon, b)$ so that this argument shows that the derivative of $P(\cdot, \vec{\sim})$ is positive at some point in $(b-\varepsilon, b)$, contradicting the fact that it must be zero on $[a, b]$. Thus we must have $b=1 / 2$.

Q.E.D.

To prove Proposition 4, we need the following preliminary result.

Lemma 2. Let $c>0$. For each $n=1,2, \ldots$ let $f^{n}:[0, c] \rightarrow[0,1], f:[0, c] \rightarrow$ $[0,1]$, and $g:[0, c] \rightarrow \boldsymbol{R}$ be twice continuously differentiable and nondecreasing, with $f^{\prime \prime}(0)=0$, and $f^{n} \rightarrow f, f^{n^{\prime}} \rightarrow f^{\prime}$, and $f^{n^{\prime \prime}} \rightarrow f^{\prime \prime}$ uniformly. Then as $n \rightarrow \infty$, 


$$
n \int_{0}^{c} g^{\prime}(x)\left(1-f^{\prime \prime}(x)\right)^{n-1} d x \longrightarrow\left\{\begin{array}{l}
\infty \text { if } f^{\prime}(0)=0 \text { and } g^{\prime}(0)>0 \\
g^{\prime}(0) / f^{\prime}(0) \text { if } f^{\prime}(0)>0 .
\end{array}\right.
$$

Proof. We first claim that

for all $\delta>0$ there exist $N$ and $\varepsilon>0$ such that if $n>N$ and $x<\varepsilon$ then $\left|f^{n^{\prime}}(x)-f^{\prime}(0)\right|<\delta$.

This follows by noting that $\left|f^{\prime \prime}(x)-f^{\prime}(0)\right| \leqq\left|f^{\prime \prime}(x)-f^{\prime}(x)\right|+\left|f^{\prime}(x)-f^{\prime}(0)\right|$, and using the uniform convergence of $f^{\prime \prime}$ to $f^{\prime}$ to bound the first term (uniformly in $x$ ), and the continuity of $f^{\prime}$ to bound the second term.

Now assume that $f^{\prime}(0)=0$ and $g^{\prime}(0)>0$. By the mean value theorem for derivatives we have $f^{\prime \prime}(x) / x=f^{n^{\prime}}(y)$ for some $0<y<x$, so by (5) we know that for any $M>0$ there exist $N$ and $\varepsilon>0$ such that if $n>N$ and $0 \leqq x<\varepsilon$ then $f^{\prime \prime}(x) / x<$ $g^{\prime}(0) / 4 M$, or $1-f^{\prime \prime}(x)>1-x g^{\prime}(0) / 4 M$; since $g^{\prime}$ is continuous, we can choose $\varepsilon$ such that we also have $g^{\prime}(x)>g^{\prime}(0) / 2$ if $x<\varepsilon$. Hence,

$$
\begin{aligned}
& n \int_{0}^{c} g^{\prime}(x)\left(1-f^{n}(x)\right)^{n-1} d x>n \int_{0}^{\varepsilon} g^{\prime}(x)\left(1-f^{n}(x)\right)^{n-1} d x \\
& \quad>n \int_{0}^{\varepsilon}\left(g^{\prime}(0) / 2\right)\left(1-x g^{\prime}(0) / 4 M\right)^{n-1} d x=2 M\left(1-\left(1-\varepsilon g^{\prime}(0) / 4 M\right)^{n}\right) .
\end{aligned}
$$

So, letting $N$ be such that it also satisfies $\left(1-\varepsilon g^{\prime}(0) / 4 M\right)^{n}<1 / 2$ if $n>N$, we have

$$
n \int_{0}^{c} g^{\prime}(x)\left(1-f^{n}(x)\right)^{n-1} d x>M \quad \text { if } n>N
$$

thus establishing the first half of the limit in the Proposition.

Now suppose $f^{\prime}(0)>0$. Then by (5) there exist $N$ and $\varepsilon>0$ such that $f^{\prime \prime}(x)>$ $f^{\prime}(0) / 2>0$ if $0 \leqq x \leqq \varepsilon$ and $n>N$, and hence

$$
\begin{aligned}
n \int_{0}^{c} g^{\prime}(x)\left(1-f^{n}(x)\right)^{n-1} d x & =\int_{0}^{\varepsilon}\left(g^{\prime}(x) / f^{\prime \prime}(x)\right) n f^{n^{\prime}}(x)\left(1-f^{\prime \prime}(x)\right)^{n-1} d x \\
& +n \int_{\varepsilon}^{c} g^{\prime}(x)\left(1-f^{\prime \prime}(x)\right)^{n-1} d x \\
& =g^{\prime}(0) / f^{\prime \prime}(0)-\left(1-f^{n}(\varepsilon)\right)^{n} g^{\prime}(\varepsilon) / f^{n^{\prime}}(\varepsilon) \\
+ & \int_{0}^{\varepsilon} h^{n^{\prime}}(x)\left(1-f^{\prime \prime}(x)\right)^{n-1} d x+n \int_{\varepsilon}^{c} g^{\prime}(x)\left(1-f^{n}(x)\right)^{n-1} d x,
\end{aligned}
$$

where $h^{n}(x)=g^{\prime}(x) / f^{n^{\prime}}(x)$. Now, since each $f^{n}$ is nondecreasing, and $f^{n^{\prime}}(x)>$ $f^{\prime}(0) / 2$ if $0 \leqq x \leqq \varepsilon$ and $n>N$, we have $f^{\prime \prime}(x)>\varepsilon f^{\prime}(0) / 2$, or $1-f^{n}(x)<1-\varepsilon f^{\prime}(0) / 2$, if $\varepsilon \leqq x \leqq c$ and $n>N$. Hence the limits of the second and fourth terms are zero. Now consider the third term, in which $h^{\prime \prime}(x)=g^{\prime \prime}(x) / f^{\prime \prime}(x)-f^{\prime \prime \prime}(x) g^{\prime}(x) /\left(f^{\prime \prime}(x)\right)^{2}$. Since $g^{\prime}, g^{\prime \prime}$, and $f^{\prime \prime}$ are continuous, they are bounded on $[0, c]$; since in addition $f^{\prime \prime \prime} \rightarrow f^{\prime \prime}$ uniformly, $\left\{f^{\prime \prime}\right\}$ is uniformly bounded on $[0, c]$. Thus the fact that $f^{\prime \prime}(x)>f^{\prime}(0) / 2$ if $0 \leqq x \leqq \varepsilon$ implies that $\left\{h^{n \prime}\right\}$ is uniformly bounded on $[0, c]$. 
Further, we have $f^{\prime \prime}(x) \geqq x f^{\prime}(0) / 2$, or $1-f^{\prime \prime}(x) \leqq 1-x f^{\prime}(0) / 2$, if $0 \leqq x \leqq \varepsilon$, so that the limit of the third term is zero. Finally, the limit of the first term is $g^{\prime}(0) / f^{\prime}(0)$, completing the proof.

Proof of Proposition 4. Since each $F^{n}$ is atomless, we can conclude, as in the proof of Proposition 3, that $P^{n}\left(z, F^{n}\right)=1 / n$ (the equilibrium payoff of each firm), or $n P^{n}\left(z, F^{n}\right)=1$, for all $z \in \operatorname{supp} F^{n}$. (We write $F^{n}=\left(F^{n}, \ldots, F^{n}\right)$, and now explicitly record the dependence of the payoff functions on $n$.) Also, since the equilibrium payoff $1 / n$ goes to zero as $n \rightarrow \infty$,

for any $z \in \operatorname{supp} C$ and any $\varepsilon>0$ there exists $N$ such that $(z-\varepsilon, z+\varepsilon) \cap$ supp $F^{n} \neq \phi$ if $n>N$

(otherwise a firm can guarantee a positive payoff by locating at $z$ ). Hence, given the continuity of $n P^{n}\left(z, F^{n}\right)$ in $z$,

$$
\lim _{n \rightarrow \infty} n P^{n}\left(z, F^{n}\right)=1 \quad \text { for all } \quad z \in \operatorname{supp} C .
$$

Now, for each $n$ we have

$$
\begin{aligned}
n P^{n}\left(z, F^{n}\right)= & n[1-C((1+z) / 2)]\left(F^{n}(z)\right)^{n-1}+n C(z / 2)\left(1-F^{n}(z)\right)^{n-1} \\
& +\int_{0}^{z} n C^{\prime}((u+z) / 2)\left(1-\left(F^{n}(z)-F^{n}(u)\right)\right)^{n-1} d u / 2 \\
& +\int_{z}^{1} n C^{\prime}((u+z) / 2)\left(1-\left(F^{n}(u)-F^{n}(z)\right)\right)^{n-1} d u / 2
\end{aligned}
$$

(see (2)). Let $a=\min \operatorname{supp} C, b=\max \operatorname{supp} C$. Obviously supp $F^{n} \subset[a, b]$ for all $n$, and if $z \in \operatorname{supp} C \cap(a, b)$ then $0<F^{\prime \prime}(z)<1$ for large enough $n$ (see (6)), so that the first two terms in (8) go to zero. Since $C(a / 2)=0$ and $C((1+b) / 2)=1$, the first two terms of $n P^{n}\left(z, F^{n}\right)$ are also zero for $z=a$ or $b$.

We can use Lemma 2 to determine the limits of the last two terms as $n \rightarrow \infty$ by defining the variables appropriately (i.e. in the first case $c=z, x=z-u, g(x)=$ $-2 C(z-x / 2)$ and $f^{n}(x)=F^{n}(z)-F^{n}(z-x)$, while in the second case $c=1-z$, $x=u-z, \quad g(x)=2 C(x / 2+z)$, and $\left.f^{n}(x)=F^{n}(x+z)-F^{n}(z)\right)$. If $F^{\prime}(z)>0$ the limits are both $C^{\prime}(z) / 2 F^{\prime}(z)$, so that $\lim _{n \rightarrow \infty} n P^{n}\left(z, F_{n}^{\prime \prime}\right)=C^{\prime}(z) / F^{\prime}(z)$; if $F^{\prime}(z)=0$ and $C^{\prime}(z)>0$ the limits are infinite. Thus from (7) we conclude that if $z \in \operatorname{supp} C$ and $C^{\prime}(z)>0$ then $F^{\prime}(z)>0$, and hence in fact $C^{\prime}(z) / F^{\prime}(z)=1$; if $C^{\prime}(z)=0$ then $F^{\prime}(z)=0$. Thus $F^{\prime}(z)=C^{\prime}(z)$ for all $z \in \operatorname{supp} C$; since $F^{\prime}(z)=C^{\prime}(z)=0$ if $z \notin \operatorname{supp} C$, we have $F=C$, completing the proof.

Proof of Proposition 5. Let $(c, F, G)$ be an equilibrium of $\Gamma^{3}(U)$. By Proposition 2(b) we can assume without loss of generality that $F$ is not pure and $\alpha(F)<c$. Clearly we must then have $\alpha(G)=\alpha(F)=a$, say (since 2's payoff is increasing on $[0, \alpha(G)))$. We can further assume without loss of generality that $\beta(F) \geqq \beta(G)$. Clearly we must have $\beta(F) \geqq c$, and either (i) $\beta(G)=\beta(F) \geqq c$ or 
(ii) $\beta(G)<\beta(F)=c$. We proceed in a number of steps, showing in (h) that (ii) is not possible.

(a) If $x \in J(F) \cap J(G)$ and $x \leqq c$ then $x=c / 3$ : Since $x \in J(F)$, we need $K_{2}(c, x, G)$ $\geqq K_{2}(c, y, G)$ for all $y$. Since $x<c$ by Lemma 1 , the cases $y=x-$ and $y=x+$ imply that $J_{G}(x)((x+c) / 4-x) \geqq 0$ and $J_{G}(x)((x+c) / 4-(c-x) / 2) \geqq 0$, or $x=c / 3$, given that $x \in J(G)$.

(b) $(\operatorname{supp} H) \cap[a, \min (\beta(G), c)]=[a, \min (\beta(G), c)]$ for $\quad H=F, G$ : Let $[a, \min (\beta(G), c)]=L$. Suppose $x, y \in \operatorname{supp} F$, but $(x, y) \cap(\operatorname{supp} F) \cap L=\phi$ for $a<x<y<\min (\beta(G), c)$. Then $K_{3}(c, t, s+\varepsilon)-K_{3}(c, t, s)$ is zero if $x<s<y$ and $t \leqq x$ and positive if $t \geqq y$, so that $K_{3}(c, F, s)$ is increasing in $s$. Hence $(x, y) \cap$ (supp $G)=\phi$, and either $x \notin \operatorname{supp} G$ or $x \in J(F)$. Reversing the roles of $F$ and $G$ establishes that $x \in J(F) \cap J(G)$ and, since $(x, y)$ is an arbitrary gap, we have $(\operatorname{supp} F) \cap L=(\operatorname{supp} G) \cap L . \quad$ Now, if $x<s<y$ then $K_{2}(c, s, G)-K_{2}(c, x, G)=$ $J_{G}(x)((c-x) / 2-(c+x) / 4)+(1-G(x))(s-x) / 2$; by (a) the first term is zero, so the expression is positive, contradicting the fact that $x \in J(F)$.

(c) If $x \in J(H)$ then $x \leqq c / 3$ if $x<\min (\beta(G), c)$, and $x \geqq c / 3$ if $x>a$, for $H=F$, $G$ : If $x \in J(F)$ and $x<\min (\beta(G), c)$, then since $[x, x+\varepsilon] \subset$ supp $G$ for some $\varepsilon>0$ by (b), we need $K_{3}(c, F, x) \leqq K_{3}(c, F, x+)$ (which is equal to the equilibrium payoff of firm 3). This implies that $x \leqq c / 3$. Similarly if $x>a$ we need $K_{3}(c, F, x)$ $\leqq K_{3}(c, F, x-)$, which implies $x \geqq c / 3$. In both cases the argument applies also to $G$.

(d) $a \geqq c / 3$ : Suppose $a<c / 3$, and let $a \leqq s<c / 3$. Then

$$
\begin{aligned}
& K_{3}(c, F, c / 3)-K_{3}(c, F, s)=J_{F}(s)\left(\frac{c-s}{2}-\frac{c+s}{4}\right) \\
& \quad+\int_{(s, c / 3)}\left(\frac{c-t}{2}-\frac{s+t}{2}\right) d F(t)+J_{F}(c / 3)\left(\frac{c+c / 3}{4}-\frac{s+c / 3}{2}\right) \\
& \quad+(c / 3-s)(1-F(c / 3)) / 2 .
\end{aligned}
$$

Now, using the fact that

$$
\int_{(s, c / 3)} t d F(t) \leqq(c / 3)\left[F(c / 3)-J_{F}(c / 3)-F(s)\right]
$$

we obtain

$$
K_{3}(c, F, c / 3)-K_{3}(c, F, s) \geqq(c-3 s)\left[3 J_{F}(s)+2(1-F(s))\right] / 12 .
$$

But now since $F$ is not pure we have $F(s)<1$ for all $a \leqq s<a+\varepsilon$ for some $\varepsilon>0$, and hence $K_{3}(c, F, c / 3)>K_{3}(c, F, s)$ for $a \leqq s<a+\varepsilon$. But this contradicts the fact that $a \in \operatorname{supp} G$. Hence $a \geqq c / 3$.

(e) $a>c / 3$ : Suppose $a \leqq c / 3$. By (d) we then have $a=c / 3$. Let $a \leqq s<t<$ $\min (\beta(G), c)$. Then making a calculation as in (d) (using (c), which implies that $J_{F}(t)=0$ and $J_{F}(s)=0$ unless $s=a=c / 3$ ), we find

$$
K_{3}(c, F, t)-K_{3}(c, F, s) \geqq[(t-s)-3(t-a) F(t)+(s+2 t-3 a) F(s)] / 2 .
$$


Now let $F(a)=1-\delta$. Since $F$ is not pure we have $\delta>0$, and since $F$ is continuous on $(a, \min (\beta(G), c))$, there exists $t^{*}$ in this interval such that $F\left(t^{*}\right) \leqq 1-5 \delta / 6$. Let $\varepsilon=\left(t^{*}-a\right) \delta / 4$. Then if $a \leqq s<a+\varepsilon$ the right-hand side of (9) when $t=t^{*}$ is at least

$$
\left[t^{*}-a-\left(t^{*}-a\right) \delta / 4-3\left(t^{*}-a\right)(1-5 \delta / 6)+2\left(t^{*}-a\right)(1-\delta)\right] / 2 \geqq\left(t^{*}-a\right) \delta / 4>0 .
$$

Hence $K_{3}\left(c, F, t^{*}\right)>K_{3}(c, F, s)$ for all $a \leqq s<a+\varepsilon$, contradicting the fact that $a \in \operatorname{supp} G$. Hence $a>c / 3$.

(f) $\beta(G)>a:$ If $\beta(G)=a$ then $K_{2}(c, a, G)=(c+a) / 4<a=K_{2}(c, a-, G)$ (using (e)), while $K_{2}(c, s, G)=(c-a) / 2<a$ for $a<s<c$, contradicting the fact that $\alpha(F)=a$.

(g) If $x \in[a, \min (\beta(G), c))$ then $x \notin J(F)$ and $x \notin J(G)$ : this follows directly from (e) and (c).

(h) $\beta(F)=\beta(G) \geqq c$ : Assume this is not so. Then $\beta(G)<\beta(F)=c$ (see the discussion preceding (a)). Because of $(\mathrm{g}), K_{2}(c, s, G)$ and $K_{3}(c, F, s)$ are continuous in $s$ on $[a, \beta(G)$ ), and hence for equilibrium must be constant there. Using a standard argument (see, for example, the solution to Problem 17 on p. 294 of Karlin [1959]) we can establish that $F$ and $G$ are differentiable on this interval. The conditions that $K_{2}(c, \cdot, G)$ and $K_{3}(c, F, \cdot)$ be constant then imply that $F$ and $G$ satisfy the same first order differential equation on $[a, \beta(G)$ ), with the same initial condition $(F(a)=G(a)=0)$. Hence $F(s)=G(s)$ if $a \leqq s<\beta(G)$. Thus, since we are assuming $\beta(G)<\beta(F)(=c)$, we must have $\beta(G) \in J(G)$. But then $K_{2}(c, \beta(G), G)-K_{2}(c, s, G)=J_{G}(\beta(G))(3 \beta(G)-c) / 4$ if $\beta(G)<s<c$. Since $\beta(G) \geqq$ $a>c / 3$, this is positive and hence supp $F \cap(\beta(G), c)=\phi$. But then $K_{3}(c, F, s)$ is increasing on $(\beta(G), c)$ (as in (b)), so we must have $\beta(G) \in J(F)$ (so that $K_{3}(c, F, \cdot$ ) jumps down at $\beta(G)$ ). But then $\beta(G) \in J(F) \cap J(G)$, and $c / 3<\beta(G)<c$, contrary to (a). Hence $\beta(G)=\beta(F) \geqq c$.

Now let $\beta(G)=\beta(F)=b$. Then by (b) we have supp $F \cap[a, c]=\operatorname{supp} G \cap$ $[a, c]=[a, c]$; symmetric arguments yield $\operatorname{supp} F \cap[c, b]=\operatorname{supp} G \cap[c, b]=$ $[c, b]$. By (c), $F$ and $G$ are atomless, except possibly at $c$. As in the proof of (h), they are also differentiable; the conditions that $K_{2}(c, \cdot, G)$ and $K_{3}(c, F, \cdot)$ be constant on $(a, c)$ imply that $G^{\prime}(t)(c-3 t)-G(t)+1=0$ and similarly for $F$. Given $F(a)=G(a)=0$ (because of (e) and (c)) we have

$$
F(t)=G(t)=1-(3 a-c)^{1 / 3}(3 t-c)^{-1 / 3} \quad \text { if } \quad a \leqq t<c .
$$

We can make similar arguments for $c<t<b$; we obtain

$$
F(t)=G(t)=(2-3 b+c)^{1 / 3}(2-3 t+c)^{-1 / 3} \quad \text { if } \quad c<t \leqq b .
$$

Thus $F$ and $G$ have an atom of the same size at $c$; since firm 3 has an atom there, the size of the atoms is zero by Lemma 1 , and $F=G$ is differentiable except possibly at $c$.

We now determine the position of $c$. First note that for equilibrium we need $K_{2}(c, c-, F)=K_{2}(c, c+, F)$ (both of which must equal the equilibrium payoff 
of firm 2). A calculation shows that this imples that

$$
\int_{a}^{b} s F^{\prime}(s) d s=3 c-2 F(c) \text {. }
$$

Now, in order for firm 1 to use the pure strategy $c, K_{1}(s, F, F)$ must be maximized at $s=c$. This requires $K_{1}^{\prime}(c-, F, F) \geqq 0$ and $K_{1}^{\prime}(c+, F, F) \leqq 0$ (where the derivative is with respect to the first argument). But if $s \neq c$ we have

$$
K_{1}^{\prime}(t, F, F)=F^{\prime}(t)\left[2 F(t)-3 t+\int_{a}^{b} s F^{\prime}(s) d s\right]+1 / 2-F(t),
$$

so letting $t \uparrow c$ and $t \downarrow c$, and using (12), we need $F(c)=1 / 2$. Now we can let $t \rightarrow c$ in (10) and (11), and use these to calculate $\int_{a}^{b} s F^{\prime}(s) d s$ in (12). The only solution of the resulting three equations is $a=5 / 24, c=1 / 2$, and $b=19 / 24$. Hence the only candidate for equilibrium is the one specified in the Proposition.

To complete the proof we need to show that this is in fact an equilibrium. It is clear that $K_{2}(1 / 2, s, F)$ increases to $K_{2}(1 / 2, a, F)=K_{2}(1 / 2, F, F)$ as $s$ increases to $a$; similarly it increases to $K_{2}(1 / 2, b, F)=K_{2}(1 / 2, F, F)$ as $s$ decreases to $b$. Hence $F$ is a best reply of firm 2 to $(1 / 2, F)$; identical arguments apply to firm 3 . It is also clear that $K_{1}(s, F, F) \leqq K_{1}(a, F, F)$ if $s \leqq a$ and $K_{1}(s, F, F) \leqq K_{1}(b, F, F)$ if $s \geqslant b$. Thus it remains to show that $K_{1}(s, F, F) \leqq K_{1}(1 / 2, F, F)$ if $a \leqq s \leqq b$. Substituting (from (10) and (11)) into (13) we can find $K_{1}^{\prime}(s, F, F)$. Analyzing its derivative we find that $K_{1}^{\prime}$ is negative from $s=a$ until some point, and then is positive until $s=1 / 2$. Thus, the only possibilities for maxima of $K_{1}(s, F, F)$ on $[a, 1 / 2]$ are $s=a$ or $s=1 / 2$. But we find that $K_{1}(a, F, F)=14 / 48$, while $K_{1}(1 / 2$, $F, F)=19 / 48$. Hence $K_{1}(s, F, F) \leqq K_{1}(1 / 2, F, F)$ for all $a \leqq s \leqq 1 / 2$. Since $F(s)=1-F(1-s)$, it is immediate that $K_{1}(s, F, F) \leqq K_{1}(1 / 2, F, F)$ for all $1 / 2 \leqq$ $s \leqq b$ also. Hence $s=1 / 2$ is a best response of firm 1 to $(F, F)$. It is easy to check that the payoffs at the equilibrium are $(19 / 48,29 / 48,29 / 48)$, completing the proof.

\section{REFERENCES}

Aumann, R. J., Y. Katznelson, R. Radner, R. W. Rosenthal and B. Weiss, "Approximate Purification of Mixed Strategies," Mathematics of Operations Research, 8 (1983), 327-341.

Dasgupta, P. And E. Maskin, "The Existence of Equilibrium in Discontinuous Economic Games, 1: Theory," Discussion Paper 82/54, International Centre for Economics and Related Disciplines, London School of Economics (March 1982a). AND — "The Existence of Equilibrium in Discontinuous Economic Games, 2: Applications," Discussion Paper 82/55, International Centre for Economics and Related Disciplines, London School of Economics (June 1982b).

Eaton, B. C. AND R. G. Lipsey, "The Principal of Minimum Differentiation Reconsidered: Some New Developments in the Theory of Spatial Competition," Review of Economic Studies, 42 (1975), 27-49.

Harsanyi, J. C., "Games with Randomly Disturbed Payoffs: A New Rationale for Mixed Strategy Equilibrium Points," International Journal of Game Theory, 2 (1973), 1-23.

-, "A Solution Theory for Noncooperative Games and its Implications for 
Cooperative Games," Working Paper CP-401, Center for Research in Management Science, University of California, Berkeley, 1977.

Hotelling, H., "Stability in Competition," Economic Journal, 39 (1929), 41-57.

KarLIN, S., Mathematical Methods and Theory in Games, Programming, and Economics, Volume II (Reading, MA: Addison-Wesley, 1959).

Lerner, A. P. AND H. W. Singer, "Some Notes on Duopoly and Spatial Competition," Journal of Political Economy, 45 (1937), 145-186.

Milgrom, P. R. AND R. J. Weber, "Distributional Strategies for Games with Incomplete Information," Discussion Paper 428R, J. L. Kellogg Graduate School of Management, Northwestern University (1981).

SHAKED, A., "Existence and Computation of Mixed Strategy Nash Equilibrium for 3-Firms Location Problem," Journal of Industrial Economics, 31 (1982), 93-96.

Simon, L. K., "Games with Discontinuous Payoffs. Part II: Application to Hotelling," draft (1984). 